\title{
Nonlinear equilibrium structure of thin currents sheets: influence of electron pressure anisotropy
}

\author{
L. M. Zelenyi ${ }^{1}$, H. V. Malova ${ }^{1,2}$, V. Yu. Popov ${ }^{3}$, D. Delcourt ${ }^{4}$, and A. S. Sharma ${ }^{5}$ \\ ${ }^{1}$ Space Research Institute, RAS, Moscow, Russia \\ ${ }^{2}$ Scobeltsyn Institute of Nuclear Physics of Moscow State University, Moscow, Russia \\ ${ }^{3}$ Faculty of Physics, Moscow State University, Vorobyevy gory, Moscow, Russia \\ ${ }^{4}$ Centre d'étude des Environnements Terrestre et Planétaires-CNRS, Saint-Maur des Fossés, France \\ ${ }^{5}$ Department of Astronomy, University of Maryland, College Park, MD 20742, USA
}

Received: 8 September 2004 - Revised: 31 October 2004 - Accepted: 1 November 2004 - Published: 23 November 2004

\begin{abstract}
Thin current sheets represent important and puzzling sites of magnetic energy storage and subsequent fast release. Such structures are observed in planetary magnetospheres, solar atmosphere and are expected to be widespread in nature. The thin current sheet structure resembles a collapsing MHD solution with a plane singularity. Being potential sites of effective energy accumulation, these structures have received a good deal of attention during the last decade, especially after the launch of the multiprobe CLUSTER mission which is capable of resolving their 3D features. Many theoretical models of thin current sheet dynamics, including the well-known current sheet bifurcation, have been developed recently. A self-consistent 1D analytical model of thin current sheets in which the tension of the magnetic field lines is balanced by the ion inertia rather than by the plasma pressure gradients was developed earlier. The influence of the anisotropic electron population and of the corresponding electrostatic field that acts to restore quasi-neutrality of the plasma is taken into account. It is assumed that the electron motion is fluid-like in the direction perpendicular to the magnetic field and fast enough to support quasi-equilibrium Boltzmann distribution along the field lines. Electrostatic effects lead to an interesting feature of the current density profile inside the current sheet, i.e. a narrow sharp peak of electron current in the very center of the sheet due to fast curvature drift of the particles in this region. The corresponding magnetic field profile becomes much steeper near the neutral plane although the total cross-tail current is in all cases dominated by the ion contribution. The dependence of electrostatic effects on the ion to electron temperature ratio, the curvature of the magnetic field lines, and the average electron magnetic moment is also analyzed. The implications of these effects on the fine structure of thin current sheets and their potential impact on substorm dynamics are presented.
\end{abstract}

Correspondence to: L. M. Zelenyi

(lzelenyi@iki.rssi.ru)

\section{Introduction}

The existence of thin current sheets (TCS) in the Earth's magnetosphere is now confirmed by many spacecraft-borne in situ experiments, including recent multi-point Cluster measurements (Runov et al., 2003a, b; Sergeev et al., 2003). TCS represent unique plasma structures similar to a collapsing MHD solution with a plane discontinuity (Syrovatsky, 1970) and thicknesses of the order of the ion Larmor radius (Mitchell et al., 1990; Pulkkinen et al., 1993, 1994; Sergeev et al., 1993, 1998; Hoshino et al., 1996; Runov et al., 2003a, b; Zelenyi et al., 2003). TCS may exhibit various profiles of current density that significantly differ from the well known bell-shaped profile of the Harris-type plasma configuration (Harris, 1962). For example, satellite measurements sometimes reveal a bifurcated ("double-humped") profile of TCS (Runov et al., 2003a, b; Baumjohann and Nakamura, 2002) or even a triple-humped profile with a current density that has a sharp peak in the center and two peaks on the sides, as well as rectangular and asymmetric shapes (Nakamura et al., 2004).

The physical mechanisms underlying the different selfconsistent TCS structures are not well understood. It should be noted that trajectories of ions and electrons within TCS are very different. While the behavior of the thermal (usually a few $\mathrm{keVs}$ ) ions is principally nonadiabatic near the equatorial plane (Büchner and Zelenyi, 1989), the electrons (having energies 5 or 6 times lower than that of ions and at least two orders of magnitude smaller gyroradii) are mostly magnetized in the current sheet (except for a very small regions of about an electron gyroradius wide, near the $\mathrm{X}$ - and $\mathrm{O}$-lines). It is shown in experimental works by Pulkkinen et al. (1994) that during the growth phase of substorm the electron pressure in magnetotail might be anisotropic $\left(p_{\|}-p_{\perp}>0\right)$.

Numerical simulations using full kinetic, hybrid, and HallMHD codes have shown that electrons may be responsible for the characteristic bifurcated structures of TCS and could carry substantial currents in the vicinity of the weak field region, i.e. X- and O-lines (Pritchett and Coroniti, 1995; 
Pritchett, 2001; Hesse et al., 1996; Birn et al., 1998; Yin and Winske, 2002; Asano, 2001, 2003). Hoshino et al. (1996) and Asano et al. (2003) developed a numerical current sheet model where the electron current dominates at the edges of the TCS due to the $\boldsymbol{E} \times \boldsymbol{B}$ drift near the X-line, thus leading to a characteristic double-humped structure of the current density profile. Greco et al. (2002) suggested that the current bifurcation may result from chaotic particle scattering due to magnetic fluctuations. Zelenyi et al. $(2002,2003)$ developed a self-consistent model where thin current sheets are gradually "deteriorated" by quasi-trapped particles due to the nonadiabatic scattering of transient ions. This process, referred to as current sheet "aging", leads to the splitting, i.e. bifurcation to a double-humped shape, within the timescale of substorm growth phase. This raises questions about the relative contributions of electrons and ions to the TCS formation. In a recent study Zelenyi et al. (2004) investigated the role of electron current using a 1D self-consistent analytical model in the limit of isotropic pressure. A detailed analysis of the results favors the ion-dependent "aging" process rather than electron drift currents as an efficient mechanism of current sheet bifurcation.

Although only one example of a "triple" peaked structure of current density has been reported up to now (Nakamura et al., 2004), it provides an important example of the variety of possible current sheet configurations. A very thin region of electron flow at the center of the current sheet embedded in a thick ion layer may be the origin of the triple-peaked current sheet. In this paper, we use a self-consistent $1 \mathrm{D} \mathrm{ki-}$ netic model of thin current sheets (Sitnov et al., 2000a; Zelenyi et al., 2000) to examine electrostatic effects in the case of anisotropic electron pressure. The problem of the electron current in 1D TCS has not been addressed so far. In Sitnov et al. (2000b), a simple approach was adopted where the influence of electrons is taken into account only to redistribute ions in the electrostatic potential arising from quasineutrality and the electron contribution to the net current was not considered. A procedure to include the electron drift in similar self-consistent numerical TCS models has been described by Peroomian et al. (2002) in a study dedicated to large-scale kinetic modeling of the magnetotail. This paper presents the results from a combination of the two approaches to modeling, viz. large scale kinetics and selfconsistent TCS structures. Such a method is valid for both the cases of isotropic and anisotropic electron pressure.

\section{Basic equations of the model}

\subsection{General assumptions}

We present here an analytical 1D self-consistent model of a thin current sheet, taking into account both ion and electron plasma populations. The GSM coordinate system, in which the $\mathrm{X}, \mathrm{Y}$ and $\mathrm{Z}$ axes are along the Sun-Earth, dawndusk and north-south directions, respectively, is used here. The current sheet is so thin in comparison with its length (along $\mathrm{Y}$ ) and width (along $\mathrm{X}$ ) that one can consider it to be homogeneous along the $\mathrm{X}$ and $\mathrm{Y}$ directions (Sitnov et al., 2000a; Zelenyi et al., 2000). Therefore we consider a simplified magnetic field $\boldsymbol{B}=B_{x}(Z), 0, B_{n}$ in which only $B_{x}(Z)$ is dependent on $Z$ and reverses sign across the $Z=0$ plane. The $Z$-component $B_{n}$ is a constant commonly referred to as the nomal component and leads to the curved field lines. Within this sheet, magnetic field line tension is balanced by the finite inertia of ions rather than by plasma pressure gradients as in the case of Harris-like equilibria. The particles, e.g. from the mantle, flow from the edges of TCS toward the neutral sheet plane $(Z=0)$. The ion population consists of transient particles moving along Speiser-type orbits (the initial Maxwellian distribution, being characterized by the thermal velocity $v_{T}$ and the bulk flow speed $v_{D}$ ). The motion of transient particles in the center of thin current sheet might be described as two simultaneous motions: fast bounce oscillations in $Z$ coordinate and slow $X$-gyration in a magnetic field component normal to a current sheet plane $B_{n}$. These two $X$ and $Z$ oscillations in a first approximation are decoupled if $B_{n} / B_{0} \ll 1$. We assume that the dynamics of the ion population is quasi-adiabatic (Whipple et al., 1986, Büchner and Zelenyi, 1989) so that the action integral $I_{z}=\frac{m}{2 \pi} \oint V_{z} d z$ is approximately conserved. We assume that $B_{n}$ component of the magnetic field has small but finite value, so that only ions are quasiadiabatic while for electrons $B_{n}$ is strong enough to magnetize electrons, therefore the cross-field motion of the electron component is described by hydrodynamic equations with anisotropic pressure tensor. The electron motion along the field lines is assumed to be fast enough to support a quasi-equilibrium Boltzmann distribution in the presence of an electrostatic potential and mirror forces. A background plasma with quasi-neutrality $n_{i} \approx n_{e}$ is also assumed. Such an approach represents a generalization of the 1D selfconsistent TCS model developed earlier (Malova et al., 2000; Sitnov et al., 2000a; Zelenyi et al., 2000). In these models the role of the electron population was not taken into account.

The quasiadiabaticity approach $I_{z} \approx$ const. is valid under the condition $\kappa=\sqrt{R_{c} / \rho_{\max }}<1$ where $\rho_{\max }$ is the maximum gyroradius of the ion, and $R_{c}$, the minimum curvature radius (see Büchner and Zelenyi, 1989). This condition holds in the magnetotail for numerous TCS observations (Pulkkinen et al., 1994). As a matter of fact, Lui (1993) and Sergeev et al. (1993) estimated the parameter $\kappa$ in the midtail to be of ther order of 0.1 for ions and above 3 for electrons. The $\kappa$ parameter of the ions is thus small enough to ensure quasi-adiabatic conditions, whereas that of electrons is large enough to consider these particles as a magnetized component of the plasma.

In general, there exists two exact integrals of the particle motion: the total particle energy $\left[W=m v^{2} / 2+e \tilde{\varphi}(z)(\tilde{\varphi}\right.$ is the electrostatic potential) and the canonical momentum $P_{y}=m v_{y}-(e / c) A_{y}(x, z)$, which is conserved due to the absence of explicit - dependence in our model. In the quasiadiabatic case, the approximate integral of "fast" motion along Z-coordinate (Sonnerup, 1971; Francfort and Pellat, 1976; Büchner and Zelenyi, 1989; Whipple et al., 1986, 
1990) is also roughly conserved. All classes of particle populations in the magnetotail (i.e. Speiser's or transient ions, quasi-trapped ions on "cucumber"-like orbits and trapped ions with "ring"-like orbits) may actually be classified according to their $I_{z}$ values (Büchner and Zelenyi, 1989). In this study, as mentioned above, we consider only ions with Speiser's orbits and neglect the currents carried by particles executing "cucumber" and "ring" orbits. The effect of these latter populations has been investigated in our previous studies (Zelenyi et al., 2002, 2003). The derivation of selfconsistent dimensionless Vlasov-Maxwell system of equations for a plasma with one active component (ions) has been described in detail by Sitnov et al. (2000a, b) and Zelenyi et al. (2000). Here we use a similar approach to obtain selfconsistent solutions for a two-component system. The main steps of this calculation are described hereinafter.

Taking into account the equations of particle motion and the conservation of the integral $P_{y}$, the quasi-adiabatic invariant $I_{z}$ may be expressed as

$$
\begin{aligned}
& I_{z}(\boldsymbol{v}, z)=2 m / \pi \int_{z_{0}}^{z_{1}}\left(v_{y}^{2}+v_{z}^{2}+2 e\left\{\tilde{\varphi}(z)-\tilde{\varphi}\left(z^{\prime}\right)\right\}\right. \\
& \left.-\left\{v_{y}+e / m c \int_{z}^{z^{\prime}} B_{x}\left(z^{\prime \prime}\right) d z^{\prime \prime}\right\}\right)^{2} d z^{\prime}
\end{aligned}
$$

with the following limits of integration (Sitnov et al., 2000b)

$$
\begin{aligned}
& v_{y} \pm \sqrt{v_{y}^{2}+v_{z}^{2}+2 e\left\{\tilde{\varphi}(z)-\tilde{\varphi}\left(z^{\prime}\right)\right\} / m}= \\
& -(e / m c) \int_{z_{0}}^{z_{0,1}} B_{x}\left(z^{\prime \prime}\right) d z^{\prime \prime} .
\end{aligned}
$$

In the case when the solution of Eq. (2) is negative, the value $z_{0}$ is set equal to 0 .

\subsection{Vlasov-Maxwell system of equations}

For the 1D TCS model that we consider, the self-consistent system of Vlasov-Maxwell equations has the following simple form:

$$
\begin{aligned}
& \frac{d B_{x}}{d z}=(4 \pi / c)\left(j_{y i}(z)+j_{y e}(z)\right) \\
& j_{y i}=(4 \pi / c) \sum_{i} e \int v_{y} f_{i}(z, v) d \boldsymbol{v} .
\end{aligned}
$$

Here, $j_{y i}$ is the total ion current in the $y$-direction, $f_{i}$ is the ion distribution function within the current sheet which is obtained by mapping the source distribution from the edges of TCS to the neutral plane using $\mathrm{Li}^{-}$ ouville's theorem. Source distribution (corresponding to large values of $z$ ) has a form of shifted Maxwellian: $f_{i} \sim \exp \left\{-\left[\left(v_{\|}(\boldsymbol{v}, I(z))-v_{D}\right)^{2}+v_{\perp}^{2}(\boldsymbol{v}, I(z))\right] / v_{T}^{2}\right\} \quad($ where $v_{T}$ is the thermal velocity, and $v_{D}$ is the drift velocity).
We then introduce the dimensionless variables $\boldsymbol{r}=\boldsymbol{R} /\left(\omega_{0} v_{D} \varepsilon^{4 / 3}\right), \zeta=z \omega_{0} / \varepsilon^{4 / 3} V_{D}, \boldsymbol{w}=\overleftarrow{v} /\left(v_{D} \varepsilon^{2 / 3}\right)$ $I=I_{z} \varepsilon^{2 / 3} \omega_{0} /\left(m_{i} v_{T}\right), \varphi=m v_{D}^{2} \varepsilon^{4 / 3} \tilde{\varphi} / 2 e, \quad \boldsymbol{E}=c \boldsymbol{E} / B_{0} v_{D} \varepsilon^{2 / 3}$ $\tilde{p}_{\perp, \|}=p_{\perp, \|} / N_{0} T_{\perp, \|_{0}} \quad \tilde{j}=J / e n_{0} v_{D} \varepsilon^{2 / 3}, \quad n=\tilde{n} / N_{0}$, $\tilde{b}_{x}=B_{x} / B_{0}, \quad \tilde{b}=B / B_{0}$, where $\boldsymbol{r}$ is a normalized distance (here, $\zeta$ is the dimensionless $z$ coordinate), $\boldsymbol{w}$ is the dimensionless particle velocity vector, $I$ is the dimensionless adiabatic invariant, $\varepsilon=v_{T} / v_{D}$ is the ion source anisotropy parameter, $\omega_{0}=e B_{0} / m_{i} c$ is the ion gyrofrequency in the magnetic field $B_{0}$ at the edges of the sheet, $b$ is the normalized $B_{x}$ component of the magnetic field, $\varphi$ is the dimensionless electrostatic potential and $j_{e}$ is the normalized electron current in the $y$ direction: $j_{e}=e N_{e} v_{D} \varepsilon^{2 / 3} \tilde{j}_{e}$. Using relation between particle magnetic moment $\mu$ and adiabatic invariant $I_{z}$ as $I_{z}=4 \pi c \mu / \varepsilon$ (Sitnov et al., 2000b) one may then rewrite the normalized ion distribution function at the right-hand side of Eq. (4) as

$$
\begin{aligned}
& f_{i}(\boldsymbol{w}, I(\zeta))= \\
& \frac{n_{0} \exp \left(-\varepsilon^{-2 / 3} I\right) \times \exp \left(\varepsilon^{-2 / 3}\left(\left[\sqrt{w_{0}^{2}+\varphi-I(\zeta)}-\varepsilon^{-2 / 3}\right]^{2}+I(\zeta)\right)\right)}{\left(\pi^{3 / 2} v_{T}^{3}\left[1+\operatorname{erf}\left(\varepsilon^{-1}\right)\right]\right)} .
\end{aligned}
$$

In dimensionless form, the Maxwell equation (3) then becomes

$$
\begin{aligned}
& \frac{d b_{x}}{d \zeta}=\frac{4 \varepsilon}{\pi^{3 / 2}}\left(\frac{v_{D}}{v_{A}}\right)^{2}\left\{\int \frac{w_{y}}{\left[1+\operatorname{erf}\left(\varepsilon^{-1}\right)\right]}\right. \\
& \left.\exp \left(\varepsilon^{-2 / 3}\left(\left[\sqrt{w_{0}^{2}+\varphi-I}-\varepsilon^{-2 / 3}\right]^{2}+I\right)\right) d^{3} w+\frac{\varepsilon \pi^{3 / 2}}{4} \tilde{j}_{e}\right\}
\end{aligned}
$$

We simplify this equation by replacing the $\zeta$-coordinate with the dimensionless vector potential $\eta=\varepsilon^{2 / 3} \int_{0}^{\zeta} b\left(\zeta^{\prime}\right) d \zeta^{\prime}$ and by integrating over the $\eta$-coordinate:

$$
\begin{aligned}
& b_{x}(\eta)=\frac{2 \sqrt{2} \varepsilon^{1 / 6}}{\pi^{3 / 4}}\left(\frac{v_{D}}{v_{A}}\right)\left\{\int _ { 0 } ^ { \eta } \left\{\int \frac{w_{y}}{\left[1+\operatorname{erf}\left(\varepsilon^{-1}\right)\right]}\right.\right. \\
& \left.\left.\exp \left(\varepsilon^{-2 / 3}\left(\left[\sqrt{w_{0}^{2}+\varphi-I}-\varepsilon^{-2 / 3}\right]^{2}+I\right)\right) d^{3} w+\frac{\varepsilon \pi^{3 / 2}}{4} \tilde{j}_{e}\right\} d \eta^{\prime}\right\}^{1 / 2}(7)
\end{aligned}
$$

The usual boundary condition $b(\infty)=1$ is used in Eq. (7).

\subsection{Electrostatic potential and electric field}

Let us now describe the calculation of the electron current $\tilde{j}_{e}$, ambipolar electrostatic field $\boldsymbol{E}$ and potential $\varphi$ in our 1D kinetic TCS model. For simplicity, we assume that the electron distribution depends upon the magnetic moment $\mu$ as $f_{e}(\mu) \sim \delta\left(\mu-\mu^{*}\right)$, i.e. we assume that the electron component represents an ensemble of particles characterized by some average value of magnetic moment $\mu^{*}$, with parallel pressure $P_{\mid}$and perpendicular pressure $P_{\perp}$ as the diagonal components of the gyrotropic pressure tensor. Based on such 
a semi-hydrodynamic description, the electron motion within TCS is given by

$m \ddot{\boldsymbol{r}}=-e\left(\boldsymbol{E}+\frac{1}{c}[\boldsymbol{v} \times \boldsymbol{B}]\right)-\frac{\nabla p_{e}}{n_{e}}-\mu \nabla B$.

We use a fluid approach to describe the electron motion across the magnetic field lines and a guiding center approximation for the parallel motion:

$m_{e} \frac{d \boldsymbol{v}_{e \perp}}{d t}=-e\left(\boldsymbol{E}_{\perp}+\frac{\left[\boldsymbol{v}_{e} \times \boldsymbol{B}\right]}{c}\right)-\frac{\nabla_{\perp} p_{e}}{n_{e}}$

$m_{e} \frac{d \boldsymbol{v}_{e \|}}{d t}=-e \boldsymbol{E}_{\|}-\frac{\nabla p_{e \|}}{n_{e}}-\mu \nabla_{\|} B$

$(-e)$ being the electron charge. The last term in Eqs. (8) and (10) is the repulsive diamagnetic force acting on the electrons with magnetic moment $\mu, p_{i j}=p_{\perp} \delta_{i j}+\left(p_{\|}-p_{\perp}\right) h_{i} h_{j}$, $\boldsymbol{h}=\boldsymbol{B} / B$ is the electron pressure tensor, and $B=\left(B_{x}^{2}+B_{z}^{2}\right)^{1 / 2}$ is the total magnetic field. Neglecting the electron inertia for the fast parallel motion and substituting $E_{\|}=-\nabla \varphi$, we obtain from Eq. (10)

$\nabla \varphi(s)=\frac{1}{e n_{e}} \nabla_{\|} p_{e}+\frac{\mu \nabla_{\|} B(s)}{e}, \varphi_{0} \equiv \varphi(\infty)=0$,

where $s$ is the curvilinear coordinate along the field line, and $n_{e}(\boldsymbol{r}, \varphi(\zeta))=n_{i}(\boldsymbol{r}, \varphi(\varsigma))=n$ as a result of quasi-neutrality. In the $1 \mathrm{D}$ case, this equation can be easily integrated if we assume an isothermal ( $T_{e}=$ const $)$ equation of state:

$p_{\| e}=n_{e} T_{\| e}$.

Note that the quantity $\tau_{\|}=T_{i} / T_{e \|}$ here becomes an important characteristic parameter. For isothermal electrons, Eq. (11) can then be rewritten in a form corresponding to the Boltzmann distribution

$\frac{n_{e}(s)}{n_{0}}=\exp \left\{\frac{e\left(\varphi(s)-\varphi_{0}\right)-\mu\left(B(s)-B_{0}\right)}{T_{e}}\right\}$.

\subsection{Electron currents}

For the cross-field particle motion, if one neglect higher order drifts, one obtains from Eq. (9)

$\boldsymbol{v}_{e \perp}=c \frac{\boldsymbol{E}_{\perp}^{\prime} \times \boldsymbol{B}}{B^{2}}$,

where

$\boldsymbol{E}_{\perp}^{\prime}=\boldsymbol{E}_{\perp}+\frac{\nabla_{\perp} p_{e}}{e n_{e}}$.

In the case of isotropic pressure the cross-field electron current has the following simple form:

$j_{e \perp}=-e n_{e} v_{e \perp}$.

In the case of anisotropic pressure, we may use a more general form of this expression (Krall and Trivelpiece, 1973). Taking into account the influence of curvature drifts that are of particular importance here, we get

$$
\begin{aligned}
& j_{e \perp}=-e n_{e} c \frac{[\boldsymbol{E} \times \boldsymbol{h}]}{B}+\frac{c}{B}\left[\boldsymbol{h} \times \nabla_{\perp} p_{\perp e}\right]+ \\
& \frac{c}{B}\left(p_{\| e}-p_{\perp e}\right)[\boldsymbol{h} \times(\boldsymbol{h} \nabla) \boldsymbol{h}], \quad \boldsymbol{h}=\frac{\boldsymbol{B}}{B} .
\end{aligned}
$$

2.5 Components of electron anisotropic pressure. Final dimensionless equations

To calculate the electron current, one must determine the electric field and pressures (both perpendicular and parallel). In principle, the parallel electron pressure can be obtained from the second Chew-Goldberger-Low (CGL) equation of state $p_{\|} B^{2} / N^{3}=$ const (Kulsrud, 1983). Really, CGL approximation cannot be applied in the narrow region near the neural plane due to very large electron curvature drifts in this region. As one can see from Eq. (17) the curvature drift is inversely proportional to the curvature radius of the magnetic field lines. Near the equatorial plane in a very narrow region, where formally calculated electron drifts become very large, CGL approximation fails and corresponding amplitude of $j_{e \perp}$ becomes erroneously large. We have used more adequate estimates based on the energy conservation arguments for electrons. This equation however is not valid in the TCS neutral layer where $B$ vanishes, and equivalently, the CGL assumption for the background plasma is violated. Instead of this equation, we use the conservation of the particle energy since we examine the equilibrium of a conservative system.

$\frac{m v^{2}}{2}+e \varphi=\frac{m v_{0}^{2}}{2} \equiv$ const,$\quad v^{2}=v_{\|}^{2}+v_{\perp}^{2}$.

With $v_{\perp}^{2}=v_{\perp 0}^{2} \frac{B(z)}{B_{0}}=q^{2} v_{0}^{2} \frac{B(z)}{B_{0}}\left(q \equiv \sin \theta=\left(\tau_{\|} /\left(\tau_{\|}^{+} \tau_{\perp}\right)\right)^{1 / 2}\right.$, where $\theta$ is the average pitch-angle of electrons), we obtain:

$p_{\|}=n m v_{0}^{2}-n m v_{\perp}^{2}=n m v_{0}^{2}\left(1-q^{2} \frac{B(z)}{B_{0}}\right)$.

Perpendicular pressure is taken from the first CGL equation of state (Chew et al., 1956)

$\frac{p_{\perp}}{n B}=$ const.

Using the above mentioned dimensionless variables, one can rewrite Eqs. $(11,17,19,20)$ as:

$\nabla_{\|} \tilde{\varphi}(\tilde{s})=\varepsilon^{2 / 3}\left[\frac{1}{\tau_{\|}} \frac{\nabla_{\|}\left\{\tilde{n}\left(1-q^{2} b(z)\right\}\right.}{\left(1-q^{2}\right) \tilde{n}}+\frac{1}{\tau_{\perp}} \nabla b\right]$
$\tilde{E}_{\perp}=-\frac{1}{2 \varepsilon^{2 / 3}} \frac{d \tilde{\varphi}}{d \tilde{r}_{\perp}} ; \tilde{E}_{\|}=-\frac{1}{2 \varepsilon^{2 / 3}} \frac{d \tilde{\varphi}}{d \tilde{s}} ; b=\left(b_{x}^{2}+b_{n}^{2}\right)^{1 / 2}$

$\tilde{j}_{e \perp}=-\tilde{n} \tilde{w}_{\perp}+\frac{1}{2 b}\left(\frac{\tilde{p}_{\| e}}{\tau_{\|}}-\frac{\tilde{p}_{\perp e}}{\tau_{\perp}}\right) \frac{[\boldsymbol{b},(\boldsymbol{b} \nabla) \boldsymbol{b}]}{b^{3}}$

$\tilde{w}_{\perp}=\frac{1}{b^{2}}\left[\tilde{E}^{\prime}, \boldsymbol{b}\right], \tilde{E}^{\prime}=\tilde{E}+\frac{1}{2 \tau_{\perp} \tilde{n}}\left[\nabla \tilde{p}_{\perp}, \boldsymbol{b}\right]$

$\tilde{p}_{\|}(z)=\tilde{n} \frac{1-q^{2} b}{1-q^{2}}$

$\tilde{p}_{\perp}=\tilde{n} b$

Here we have reduced the problem to four dimensionless parameters that characterize the TCS properties, viz. $\varepsilon=\frac{v_{T i}}{v_{D i}}$, $\tau_{\|}=\frac{T_{i}}{T_{e \|}}, \tau_{\perp}=\frac{T_{i}}{T_{e \perp}}, b_{n}=\frac{B_{n}}{B_{0}}$. 


\subsection{Method of calculations}

The system of Eqs. (7), (21)-(24) together with boundary conditions imposed on the magnetic field and on the electrostatic potential fully describes our 1D semi-fluid equilibrium model of self-consistent current sheet. A set of numerical solutions of these equations can be obtained using a double iteration process in which the anisotropic electron current is not considered in the first step. The ion current, density and magnetic field are calculated using the standard iteration process (Eq. 7 with $\tilde{j}_{e}=0$ ). To recalculate the electrostatic potential, the ion density and quasi-neutrality condition are then substituted into Eq. (21). The electrostatic potential is thus used to calculate the electron current using Eq. (22). This iteration procedure is then repeated taking into account the electron current in Eq. (7), until the desired convergent solution is reached (usually after 7-10 iteration steps).

\section{Results}

By solving the system of Eqs. (7), (21)-(23) with corresponding boundary conditions, the self-consistent profiles of partial and total current densities, plasma density, electric and magnetic fields as well as electrostatic potential are obtained. For clarity, we show here the characteristic profiles of TCS as a function of three essential parameters of the system (e.g. $\left.b_{n}, \tau_{\perp}, \tau_{\|}\right)$. Another parameter $\varepsilon=1$, will be considered below for values typical of the Earth's magnetotail. Figure 1a shows the TCS current density for different initial values of $b_{n}$ and for the case of anisotropic electron pressure. The fine structure of this profile of the current density is determined by the principal difference between the motion of ions and electrons inside TCS. Ions (with the Larmor radius about CS thickness) are demagnetized near the neutral plane and are moving along open-ended orbits experiencing meandering type of motion near the neutral plane. The characteristic thickness of a "pure" ion current sheet (i.e. sheet with electrons serving only a cold neutralizing background) is about ion Larmor radius, and its profile is determined by non-harmonic oscillations of nonadiabatic ions in the region of strong magnetic field inhomogeneity. Otherwise electrons (with Larmor radius 50-100 times smaller) usually are magnetized in the current sheet, and they move along drift orbits. Corresponding drift electron currents are $[\boldsymbol{E} \times \boldsymbol{B}]$, gradient and curvature ones. The third kind of particle drift depending on the anisotropy of electron pressure absolutely predominates in a narrow region in the very center of the current sheet, where the curvature radii of the magnetic field lines are very small. The thickness of this region is proportional approximately to $L\left(B_{n} / B_{0}\right)^{4 / 3}$ (Zelenyi et al., 2000), i.e. it has relatively narrow scale in comparison with characteristic thickness of the whole sheet $\left(L \sim \rho_{0 i}\right)$. Anisotropic electrons in Fig. 1a support a very narrow current maximum in the vicinity of the neutral plane, which is embedded inside a more thicker ion current. This peak is entirely due to the electron curvature drift (last term on the RHS of Eq. 17) in

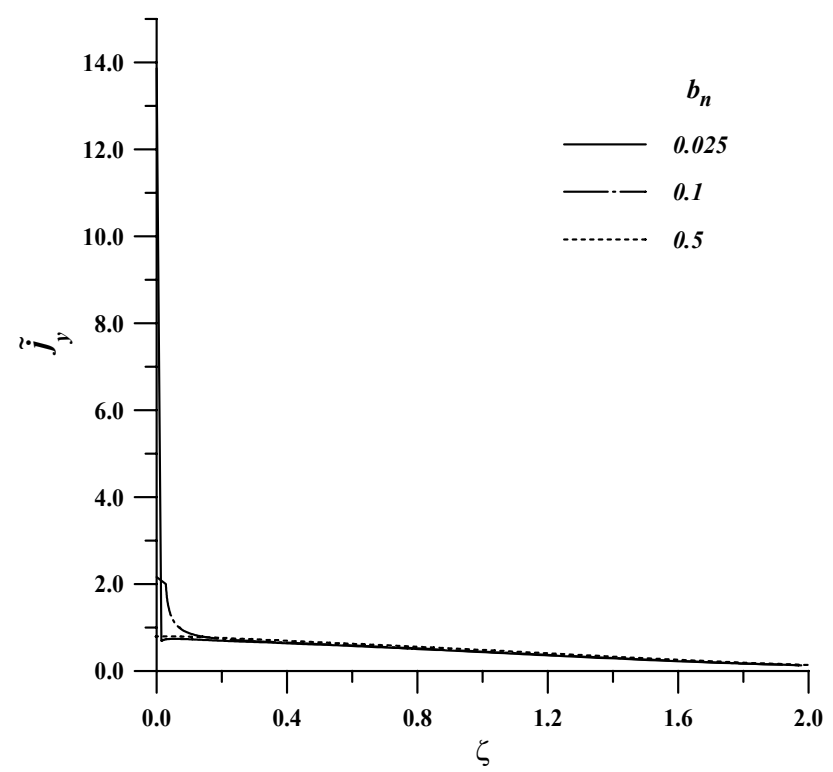

(a)

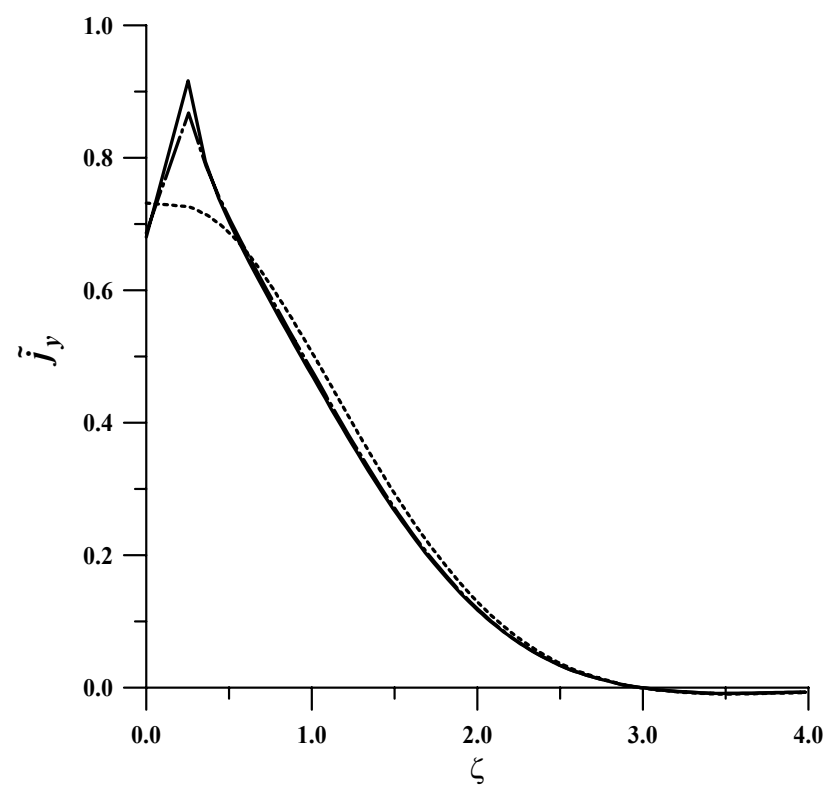

(b)

Fig. 1. Profiles of the dimensionless current densities in TCS for different values of parameter $b_{n}=0.025$ (solid line), 0.1 (dasheddotted line), 0.5 (dotted line) as functions of dimensionless $\mathrm{z}$ coordinate $\zeta$ in (a) anisotropic case of electron pressure, (b) isotropic case (Zelenyi et al., 2004).

the region of a large magnetic field line curvature. A remarkably strong $b_{n}$ dependence of the current maximum in the neutral plane can be seen. For comparison, the influence of isotropic electrons on the TCS structure when the essential electron contribution is given by $\boldsymbol{E} \times \boldsymbol{B}$ and gradient particle drifts (first and second terms on the RHS of Eq. 17) is shown in Fig. 1b. These isotropic electron effects lead to a current sheet splitting and the appearance of a characteristic bifurcated (double-humped) structure. Figure 2a shows 


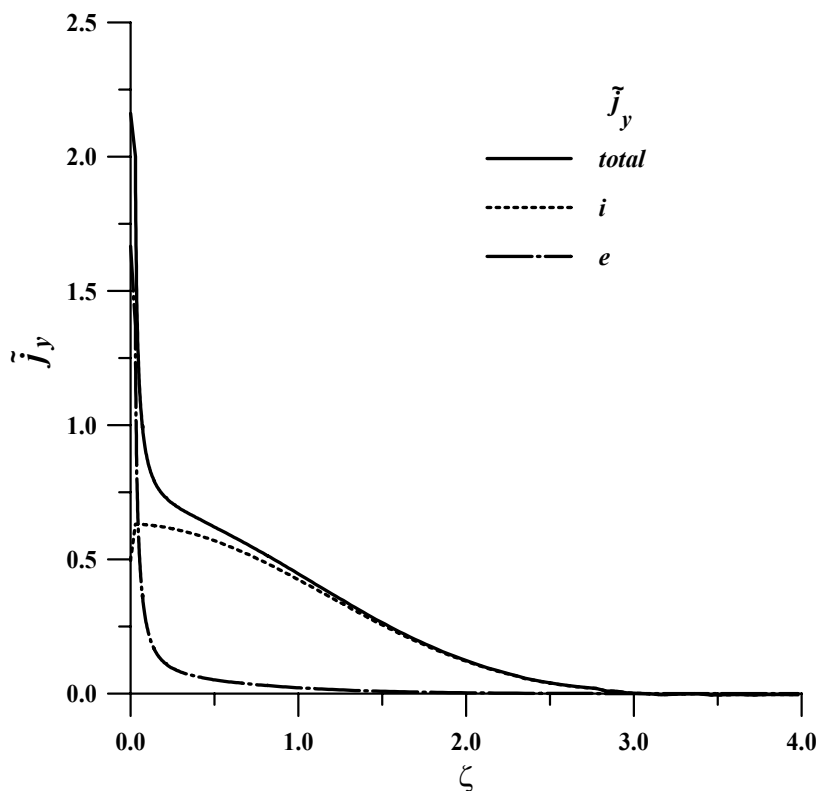

(a)

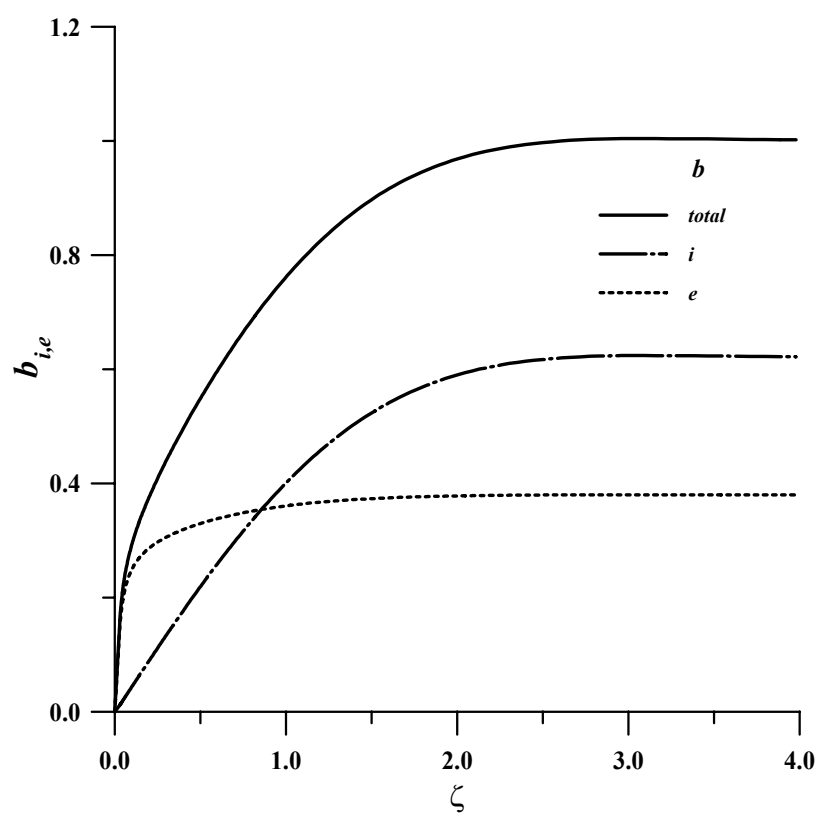

(b)

Fig. 2. Profiles of the dimensionless characteristics of TCS show contributions of (a) partial electron and ion current densities and (b) corresponding magnetic fields for fixed values $b_{n}=0.1$ and $\varepsilon=1$.

the internal structure of a total TCS current, i.e. the relative ion and electron contributions to the current density. The corresponding magnetic field profiles are shown in Fig. 2b. One can see that the characteristic steepened structure of the magnetic field profile is governed by ansiotropic electrons whereas the general large-scale structure is formed by current-carrying ions. For realistic $b_{n}$ values, the curvature drift is much larger than $\boldsymbol{E} \times \boldsymbol{B}$ and gradient drifts of electrons in the isotropic case (Fig. 1b). One can see that the maximum of electron current in the very center of the sheet

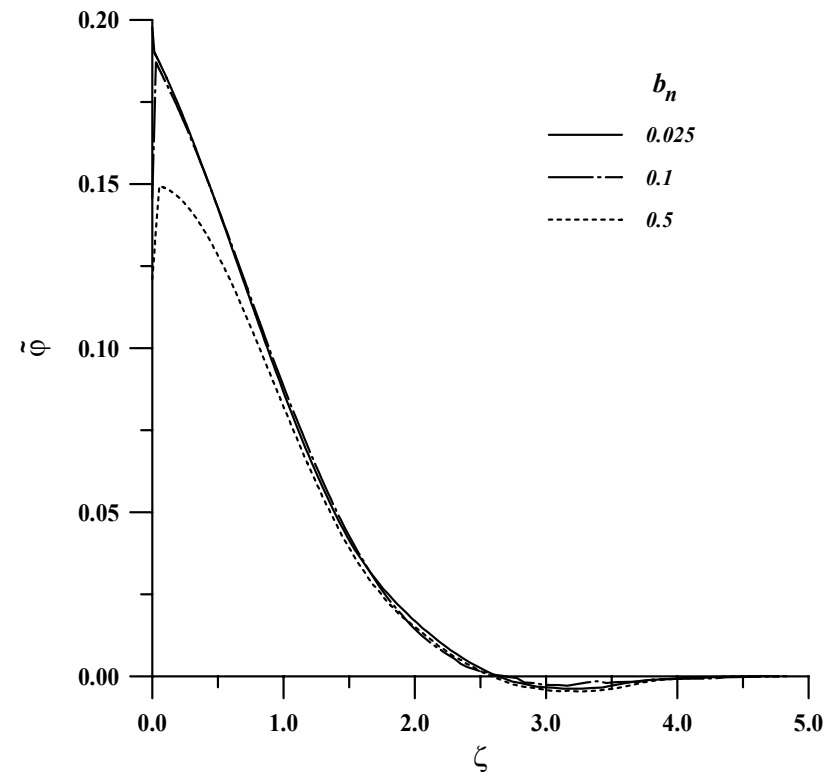

Fig. 3. Profiles of the electrostatic potential for different $b_{n}$ as functions of coordinate $\zeta$.

varies roughly in an inverse proportion to the value of normal magnetic field component $b_{n}$, since this component controls the curvature radius of the field lines. Corresponding profiles of the electrostatic potential are shown in Fig. 3. It shows that in general electrostatic potential is positive due to current carrying ions, which become temporarily trapped in the vicinity of CS. The peculiarities of potential profiles are due to the difference between electron and ion dynamics. Magnetized electrons move along closed large-scale figure eight-like drift orbits gyrating around magnetic field lines and quickly moving along them (especially in a very center of the current sheet). Thus electron density should have a depression in a central region. The requirement of charge neutrality leads to the appearance of an ambipolar electric field, which favors the net plasma rarefaction near the neutral plane. As a result ions might be pushed out from the center of current sheet to its edges, thus generating the local minimum of total plasma density in the center. As one can see from Fig. 3 this effect is more noticeable for larger $B_{n}$ values when the influence of magnetized electron motion is stronger. Figure 4 shows corresponding profiles of the magnetic field. One can see a substantial steepening of the magnetic field profile near the neutral plane. As we have mentioned above electron curvature drift currents in the center of the sheet are responsible for this effect. Figure 5 presents the profiles of TCS current density as a function of the ratios $\tau_{I I}=T_{i} / T_{e I I}=\tau_{\perp}=T_{i} / T_{e \perp}$. Figure 5 shows that peak amplitude of electron current is inversely proportional to the ratio $T_{i} / T_{e}$. For a typical value of the ion to electron temperature ratio in the magnetotail $\tau_{I I, \perp} \sim 5$, one can clearly see a multilayer structure, viz., electron currents are embedded within a much broader region where the ion contribution is dominant. 


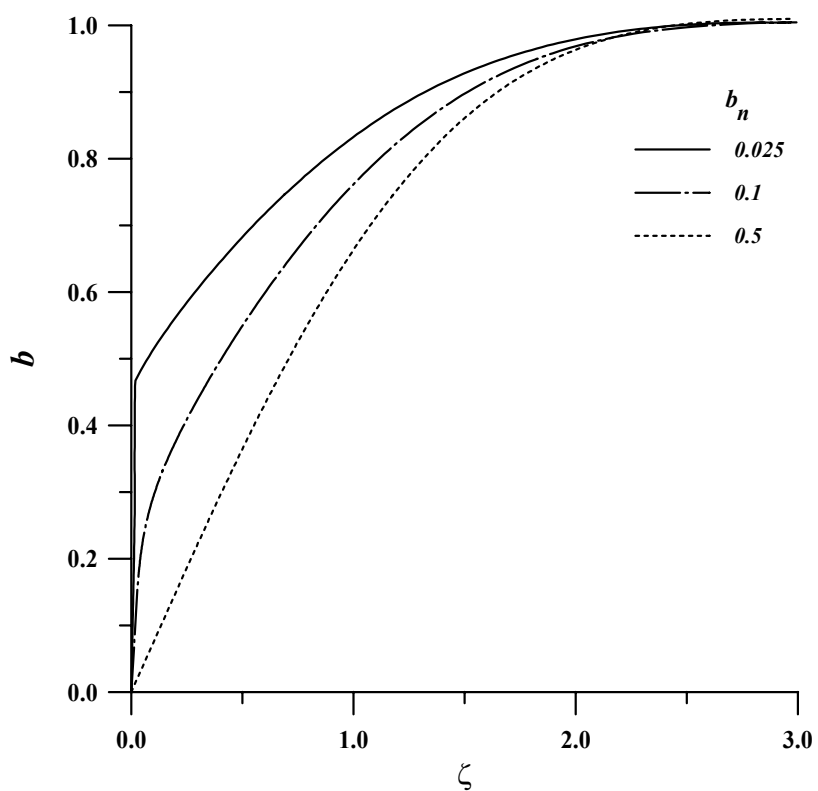

Fig. 4. Self-consistent profiles of the dimensionless magnetic field in TCS for different values of parameter $b_{n}$, corresponding to Fig. 1a.

\section{Discussion}

The results of our model show that there exists a class of equilibrium solutions of 1D self-consistent TCS configuration where anisotropic electrons provide a very characteristic contribution to the overall current structure. It has been shown earlier that the electrons may carry a significant part of the cross-tail current and lead to a bifurcated structure of the current density profile (Asano, 2001, 2003). In a more general case of electrons with anisotropic pressure, the TCS structure exhibits another characteristic feature. In the case of isotropic electrons, the magnetic field profile is flattened in the center of the sheet (Zelenyi et al., 2004), whereas in the anisotropic case it is steeper in this region. Important that anisotropy becomes a key word for the physics of such sheets and this physic is very different from that one meets dealing with isotropic Harris-type sheets. Interesting that even the very early pioneering work by Coppy and Rosenbluth (1968) empasized the critical role anisotropy could play in destabilization of such systems.

Recent CLUSTER data show (Nakamura et al., 2004) that thin current sheets in the Earth's magnetotail during geomagnetically active periods become very dynamical and that the shape of the current density profile may substantially evolve with time, viz. exhibit double or even multiple peaks. It is not clear whether such structures represent successive phases of current sheet evolution or appear separately under specific external and/or internal conditions. A few theoretical models have attempted to explain such complicated current sheet structures. Hoshino et al. (1996) and Asano et al. (2003) suggested that the splitting of the cross-tail current is due to the influence of Hall effects. On the other hand, Zelenyi et

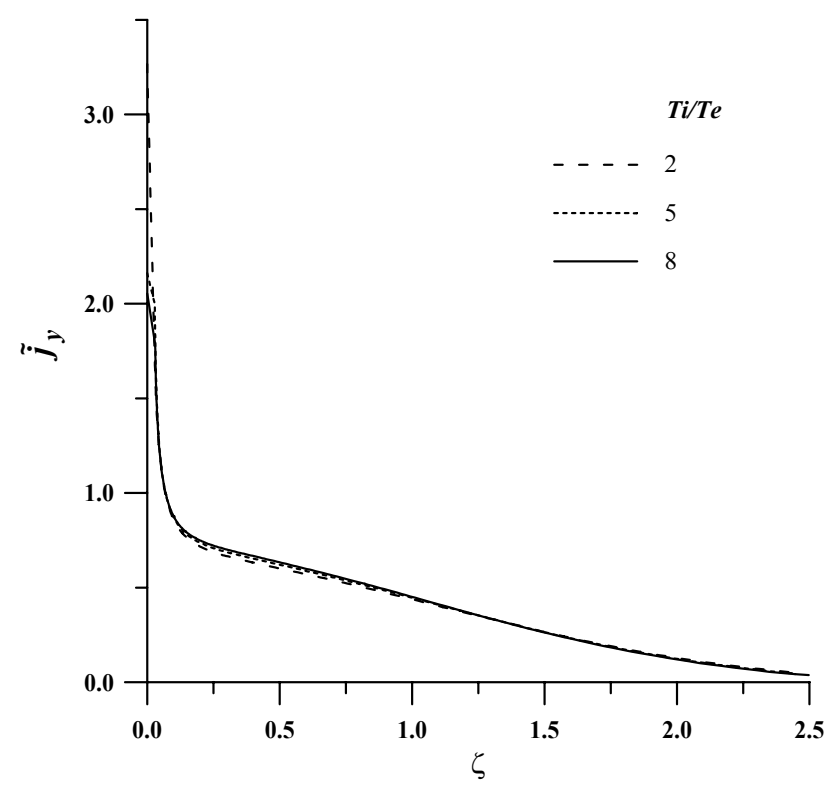

Fig. 5. Profiles of TCS current densities as a functions of coordinate $\zeta$ for different values of parameters $\tau_{\perp}=\tau_{I I}=2,5,8$.

al. (2002) proposed that the current sheet bifurcation results from accumulation of quasi-trapped plasma. Also, Greco et al. (2002) discussed the implications of ion scattering at low frequency magnetic field fluctuations and showed that this may lead to current bifurcation as well. Sitnov et al. (2003) considered small anisotropies in ion temperature in Harrislike equilibria and obtained bifurcated structures for specified parameter ranges. Our model allows to estimate amplitudes of a large scale electrostatic field building up in a sheet to keep it charge neutral. Of course, there are other mechanisms supporting formation of electric field across the current sheet. Daughton et al. (2004) considered scattering of particles at low frequency LHD fluctuations at the edges of the Harris- type current sheet (where density gradients reach maximum). This scattering provides cross-field mobility to particles and (in absence of $B_{n}$ ) might result to some effects of the type we considered above: ion leakage from the central region and appearance of the electrostatic field restoring charge neutrality. In a future in the dynamic anisotropic current sheet model it might be interesting to study the combined action of both effects

So far the model presented here, which explicitly takes into account electron curvature drifts, is the only model that can explain more sophisticated cross-tail current profiles with triple peaks. Recent multi-spacecraft observations have shown that the cross-tail current profiles exhibit a high degree of complexity and variability and it is expected that CLUSTER and other upcoming missions will provide the detailed measurements needed to resolve important physics issues.

Acknowledgements. The authors are grateful to V. Sergeev, W. Baumjohann, R. Nakamura and A. Runov for their profound interest in our work and very fruitful discussions of theoretical results and Cluster findings. This work was supported by the Russian 
Foundation of Basic Research grants 02-02-16003, 03-02-16967, grant of Council of the President of the Russian Federation for Support of Leading Scientific Schools HIII-1739.2003.2, INTAS grant 03-51-3738 and NASA grant NNG04GE37G.

Edited by: J. Büchner

Reviewed by: I. Silin and another referee

\section{References}

Asano, Y.: Configuration of the thin current sheet in substorms, Ph.D. thesis, Univ. Tokyo, 2001.

Asano, Y., Mukai T., Hoshino, M., Saito, Y., Hayakawa, H., and Nagai, T.: Evolution of the thin current sheet in a substorm observed by Geotail, J. Geophys. Res., 108, doi:10.1019/2002JA009785, 2003.

Baumjohann, W. and Nakamura, R.: What is Cluster telling us about magnetotail dynamics, Proceedings of 34th COSPAR scientific assembly, Houston, TX, USA, 10-19 October, 2002, Proc. D3.1-0027-02, 1-9, 2002.

Birn, J., Hesse, M., and Schindler, K.: MHD Simulations of Magnetotail Dynamics, J. Geophys. Res., 101, 12 939-12 954, 1996.

Büchner, J. and Zelenyi, L. M.: Regular and chaotic charged particle motion in magnetotaillike field reversals: 1 . Basic theory of trapped motion, J. Geophys. Res., 94, 11 821-11 842, 1989.

Chew, G. F., Goldberger, M. L., and Low, F. E.: The Boltzmann equation and the one-fluid hydromagnetic equations in the absence of particle collisions, Proc. Roy. Soc. (London), A236, 112,1956

Coppi, B. and Rosenbluth, M. N.: Model for the Earth's Magnetic Tail, "The Stability of Plane Plasmas", ESROSP-36 (1968), Proc. ESRIN Study Group, Rome, Italy (3-8 December 1967), edited by Schindler, K., European Space Research Institute, Rome, Italy, 1, 1968.

Daughton W., Lapenta, G., and Ricci, P.: Nonlinear evolution of the Lower-hybrid drift instability in a current sheet, Geophys. Res. Lett., in press, 2004.

Francfort, P. and Pellat, R.: Magnetic merging in collisionless plasmas, Geophys. Res. Lett., 3, 433-436, 1976.

Greco, A., Taktakishvili, A. L., Zimbardo, G., Veltri, P., and Zelenyi, L. M.: Ion dynamics in the near-Earth magnetotail: magnetic turbulence versus normal component of the average magntic field, J.Geophys. Res., 107, 1267, doi:10.1029/2002JA009270, 2002.

Harris, E. G: On a Plasma Sheath Separating Regions of Oppositely Directed Magnetic Fields, Nuovo Chimento, 23, 115-121, 1962.

Birn, J., Hesse, M., and Schindler, K.: MHD Simulations of Magnetotail Dynamics, J. Geophys. Res., 101, 12 939-12 954, 1996.

Hesse, M., Winske, D., Kuznetsova, M. M., Birn, J., and Schindler, K.: Hybrid modeling of the formation of thin current sheets in magnetotail configurations, J. Geomagn. Geoelectr., 48, 749763, 1996.

Hoshino, M., Nishida, A., Mukai, T., Saito, Y., and Yamamoto, T.: Structure of plasma sheet in magnetotail: double-peaked electric current sheet, J. Geophys. Res., 101, 24 775-24 786, 1996.

Krall, N. A. and Trivelpiece, A. W.: Principles of Plasma Physics, McGraw Hill, Inc., New York, 1993.

Kulsrud, R. M.: MHD description of plasma, Basic Plasma Physics, Vol. I, edited by Galeev, A. A. and Sudan, R. N., North-Holland Pub., Amsterdam, 1983.
Lui, A. T. Y.: Inferring global characteristics of current sheet from local measurements, J. Geophys. Res., 98, 13 423-13 427, 1993.

Malova, H. V., Sitnov, M. I., Zelenyi, L. M., and Sharma, A. S. Self-consistent model of 1D current sheet: the role of drift, magnetization and diamagnetic currents, in Proceedings of Chapman Conference: Magnetospheric Current Systems, 118, 313-322, 2000.

Mitchell, D. G., Williams, G. J., Huang, C. Y., Frank, L. A., and Russell, C. T.: Current carriers in the near-Earth cross-tail current sheet during substorm growth phase, Geophys. Res. Lett., 17, 583-586, 1990.

Nakamura, R., Baumjohann, W., Runov, A., Asano, Y., Balogh, A., and Reme, H.: Thin current sheets measured by Cluster at 200 $\mathrm{km}$ tetrahedron scale. The Second Workshop on Thin Current Sheets 19-21 April, 2004, College Park, Maryland, USA, http: //www.glue.umd.edu/ $\sim$ sitnov/TCS/tcs_1_files/online.htm, 2004.

Peroomian, V., Zelenyi, L.M., and Schriver, D.: Imprints of smallscale nonadiabatic particle dynamics on large-scale properties of dynamical magnetotail equilibria, COSPAR, Publ. Elsevier Science Ltd., UK, Adv. Sp. Res., 12, 2657-2662, 2002.

Pritchett, P. L. and Coroniti, F. V.: Formation of thin current sheets during plasma sheet convection, J. Geophys. Res., 100, $23551-$ 23 565, 1995.

Pritchett, P. L.: Geospace Environment Modeling magnetic reconnection challenge: Simulations with a full particle electromagnetic code, J. Geophys. Res., 106, 3783-3798, 2001.

Pulkkinen, T. I., Baker, D. N., Owen, C. J., Gosling, J. T., and Murthy, N.: Thin current sheets in the Deep Geomagnetotail, Geophys. Res. Lett., 20, 2427-2430, 1993.

Pulkkinen, T. I., Baker, D. N., Mitchell, D. G., McPherron, R. L., Huang, C. Y., and Frank, L A.: Thin Current Sheets in the Magnetotail During Substorms: CDAW 6 Revisited, J. Geophys. Res., 99, 5793-5803, 1994.

Runov, A., Nakamura, R., Baumjohann, W., Zhang, T. I., and Volverk, M.: Cluster observation of a bifurcated current sheet, Geophys. Res. Lett., 30, 1036, doi:10.1029/2002GL016136, 2003a.

Runov, A., Nakamura, R., Baumjohann, W., Treumann, R. A., Zhang, T. L., Volwerk, M., Vörös, Z., Balogh, A., Glassmeier, K.-H., Klecker, B., Rème, H., and Kistler, L.: Current sheet structure near magnetic X-line observed by Cluster, Geophys. Res. Lett., 30, 1579, 10.1029/2002GL016730, 2003b.

Sergeev, V. A., Mitchell, D. G., Russell, C. T., and Williams, D. J.: Structure of the tail plasma/current sheet at $11 \mathrm{Re}$ and its changes in the course of a ubstorm, J. Geophys. Res., 98, 17345-17365, 1993.

Sergeev, V. A., Angelopoulos, V., Carlson, C., Sutcliffe, P.: Current sheet measurements within a flapping plasma sheet, J. Geophys. Res., 103, 9177-9187, 1998.

Sergeev, V. A., Runov, A., Baumjohann, W., Nakamura, R., Zhang, T. L., Volwerk, M., Balogh, A., Rème, H., Sauvaud, J.-A., André, M., and Klecker, B.: Current sheet flapping 15 motion and structure observed by Cluster, Geophys. Res. Lett., 30, 1327, 10.1029/2002GL016500, 2003.

Sitnov, M. I., Zelenyi, L. M., Malova, H. V., and Sharma, A. S.: Thin current sheet embedded within a thicker plasma sheet: Selfconsistent kinetic theory, J. Geophys. Res., 105, 13 029-13 043, 2000a.

Sitnov, M. I., Zelenyi, L. M., Sharma, A. S., and Malova, H. V.: Distinctive features of forced current sheets: electrostatic effects, Proc. Int. Conf. Substorm-5, St. Petersburg, Russia, 16-20 May, 197-200, 2000b. 
Sitnov, M. I., Guzdar, P. N., and Swisdak, M.: A model of the bifurcated current sheet, Geophys. Res. Lett., 30, 13, 45, doi:10.1029/2003GL017218, 2003.

Sonnerup, B. U. Ö.: Adiabatic particle orbits in a magnetic null sheet, J. Geophys. Res., 76, 8211-8222, 1971.

Syrovatsky, S. I.: Particle Acceleration and Plasma Ejection from the Sun The Sun. Part 1 of Solar-Terrestrial Physics/1970, Comprising the Proceedings fo the International Symposium on Solar-Terrestrial Physics Held in Leningrad, USSR, 12-19 May 1970, edited by de Jager, C. and Dyer, E. R., Dordrecht, The Netherlands, D. Reidel Publishing Company, Astrophysics and Space Science Library, 29, 119, 1970.

Whipple, E. C., Northrop, T. G., and Brimingham, T. J.: Adiabatic conditions, charged particles, Earth magnetosphere, electric fields, magnetic fields, particle motion, field strength, magnetic moments, plasma drift, shear layers, shock waves, J. Geophys. Res., 91, 4149-4156, 1986

Whipple, E. C., Rosenberg, M., and Brittnacher, M.: Magnetotail acceleration using generalized drift theory: A kinetic merging scenario, Geophys. Res. Lett., 17, 1045-1048, 1990.
Yin, L. and Winske, D.: Simulations of current sheet thinning and reconnection, J. Geophys. Res., 107, 1485, doi:10.1029/2002JA009507, 2002.

Zelenyi, L. M., Sitnov, M. I., Malova, H. V., and Sharma, A. S.: Thin and Superthin Ion Current Sheets, Quasiadiabatic and Nonadiabatic Models, Nonl. Proc. Geophys., 7, 127-139, 2000, SRef-ID: 1607-7946/npg/2000-7-127.

Zelenyi, L. M., Delcourt, D. C., Malova, H. V., and Sharma, A. S.: "Aging" of the magnetotail thin current sheets, Geophys. Res. Lett., 29, doi:10.1029/2001GL013789, 2002.

Zelenyi, L. M., Malova, H. V., and Popov, V. Yu.: Bifurcation of thin current sheets in the Earth's magnetosphere, JETP Letters (Transl. from Russian), 78, 296-299, 2003.

Zelenyi, L. M., Malova, H. V., Popov, V. Yu., Delcourt, D. C., and Sharma, A. S.: Role of electrostatic effects in thin current sheets", Nato Science Series: II: Mathematics, Physics and Chemistry, Magnetospheric Response to Solar Activity, edited by Safrankova, J. and Richardson, J. D., Kluwer Academic Publishers, 275-288, 2004. 